\title{
Assemblages d'algues et relations avec quelques paramètres environnementaux dans deux sites marécageux de l'Ouest-Cameroun
}

\section{Algae assemblages and relationships with some environmental parameters in two wetlands sites in western Cameroon}

\author{
Victor François Nguetsop, Théophile Fonkou, Martin Lekeufack et Jonas Yves \\ Pinta
}

Volume 22, numéro 1, 2009

URI : https://id.erudit.org/iderudit/019821ar

DOI : https://doi.org/10.7202/019821ar

Aller au sommaire du numéro

Éditeur(s)

Université du Québec - INRS-Eau, Terre et Environnement (INRS-ETE)

ISSN

0992-7158 (imprimé)

1718-8598 (numérique)

Découvrir la revue

Citer cet article

Nguetsop, V. F., Fonkou, T., Lekeufack, M. \& Pinta, J. Y. (2009). Assemblages d'algues et relations avec quelques paramètres environnementaux dans deux sites marécageux de l'Ouest-Cameroun. Revue des sciences de l'eau / Journal of Water Science, 22(1), 15-27. https://doi.org/10.7202/019821ar

\section{Résumé de l'article}

Les échantillons destinés à l'étude des algues ont été prélevés par grattage sur différents types de substrats et par expression de macrophytes immergées dans deux sites marécageux situés dans la région Ouest du Cameroun. Les observations au microscope optique ont permis d'observer les organismes encore vivants dans leur déplacement et de noter des détails qui pourraient disparaître du fait de la fixation. Les comptages ont été faits suivant des transects verticaux choisis au hasard sur la lame jusqu'à obtention de 200 individus au moins. Les données floristiques ont été ensuite soumises à une Analyse Factorielle des Correspondances (AFC). Les résultats obtenus montrent que la répartition des espèces dans l'espace semble être contrôlée, principalement, par la pollution organique. La variabilité spatiale de cette pollution organique pourrait être expliquée par la direction privilégiée des polluants organiques due aux courants d'eau, ou par une inégale distribution dans l'espace des différentes espèces de macrophytes. Le mélange des espèces de préférences écologiques différentes dans le même échantillon, de même que la contradiction entre l'écologie de certaines espèces connues de la littérature et celle déduite des paramètres chimiques mesurés, pourraient être attribués à la variabilité à courte échelle de temps des conditions physico-chimiques, en relation avec les caractéristiques du climat local. 


\title{
ASSEMBLAGES D'ALGUES ET RELATIONS AVEC QUELQUES PARAMĖTRES ENVIRONNEMENTAUX DANS DEUX SITES MARÉCAGEUX DE L'OUEST-CAMEROUN
}

\author{
Algae assemblages and relationships with some environmental parameters in two \\ wetlands sites in western Cameroon
}

Victor Francois NGUETSOP *, ThÉophile FONKOU, MARTIN LEKEUFACK et JonAs Yves PINTA

Laboratoire de Botanique Appliquée (LABOA), Département de Biologie végétale BP 67, Dschang, Cameroun

Reçu le 14 novembre 2006, accepté le 31 mars 2008

\section{RÉSUMÉ}

Les échantillons destinés à l'étude des algues ont été prélevés par grattage sur différents types de substrats et par expression de macrophytes immergées dans deux sites marécageux situés dans la région Ouest du Cameroun. Les observations au microscope optique ont permis d'observer les organismes encore vivants dans leur déplacement et de noter des détails qui pourraient disparaître du fait de la fixation. Les comptages ont été faits suivant des transects verticaux choisis au hasard sur la lame jusqu'à obtention de 200 individus au moins. Les données floristiques ont été ensuite soumises à une Analyse Factorielle des Correspondances (AFC). Les résultats obtenus montrent que la répartition des espèces dans l'espace semble être contrôlée, principalement, par la pollution organique. La variabilité spatiale de cette pollution organique pourrait être expliquée par la direction privilégiée des polluants organiques due aux courants d'eau, ou par une inégale distribution dans l'espace des différentes espèces de macrophytes. Le mélange des espèces de préférences écologiques différentes dans le même échantillon, de même que la contradiction entre l'écologie de certaines espèces connues de la littérature et celle déduite des paramètres chimiques mesurés, pourraient être attribués à la variabilité à courte échelle de temps des conditions physicochimiques, en relation avec les caractéristiques du climat local.

Mots clés : Algues, périphyton, variabilité spatiale, marécages, Cameroun.

\begin{abstract}
Samples for algal analysis were obtained through scraping or squeezing plant parts or other objects collected in the waters of two wetland sites in western Cameroon. Microscopic observations were made to identify living organisms, and to note their movement and other details that could disappear
\end{abstract}

*Auteur pour correspondance :

Téléphone: +23799551497

Courriel : vfnguetsop@yahoo.fr 
with fixation. Counts of at least 200 individuals per sample were then made on fixed samples following vertical transects randomly chosen on the slide. Identifications were made using published keys in reference documents. Floristic data obtained were submitted to Factorial Analysis. Results obtained showed that the spatial distribution of species is primarily controlled by organic pollution. The spatial variability of the organic pollution could be explained by favoured directions of water movement transporting organic pollutants, or by an irregular spatial distribution of different species of macrophytes. Occurrence in given samples of species with different ecological preferences, and the incompatibility between the species ecology known from the literature and that deduced from our field parameters, could be attributed to the short-term variability of physicochemical parameters, in relation to the local climate.

\section{Key words: Algae, periphyton, spatial variability, wetlands, Cameroon.}

\section{INTRODUCTION}

En Afrique équatoriale, les recherches dans le domaine de la biologie végétale se sont le plus souvent intéressées à l'étude des écosystèmes forestiers. En effet, pour des raisons diverses, les recherches concernant les forêts sont celles qui bénéficient d'importants financements. Les scientifiques ne portent que très peu d'intérêt aux écosystèmes limniques. Pourtant, les algues sont très étudiées dans d'autres régions du monde pour diverses applications; par exemple, elles sont utilisées dans le diagnostic de la noyade en médecine légale (LUDES et COSTE, 1996) et dans l'évaluation de la qualité des eaux (PRYGIEL et COSTE, 1993). Elles sont fortement utilisées pour évaluer de façon plus ou moins précise les paramètres physico-chimiques de l'eau tels que le pH (BATTARBEE et CHARLES, 1986; CHARLES et al., 1991), la pollution (SLADECEK, 1986), la salinité (GASSE, 2002; SERVANT-VILDARY et ROUX, 1990), la bathymétrie (BARKER et al., 1994; BRUGAM et al., 1998) etc. Certains taxons d'algues sont capables de se fossiliser après la mort des cellules en raison de certaines caractéristiques biologiques particulières; elles peuvent ainsi permettre de reconstruire les paléoenvironnements aquatiques dans leurs sites de dépôt. Par exemple les travaux de STAGER et al. (1999) et de NGUETSOP et al. (2004), focalisés sur l'étude des diatomées actuelles et fossiles des lacs Bambili et Ossa dans l'Ouest-Cameroun, ont permis de déceler les fluctuations du bilan précipitation minus évaporation (P-E) et de déduire le fonctionnement de l'ITCZ (zone de convergence intertropicale) dans cette région au cours de l'Holocène récent. Il est donc important d'intensifier l'étude des algues dans les latitudes équatoriales de l'Afrique, afin d'accéder à la biodiversité des écosystèmes limniques et préciser l'écologie des espèces qui y sont présentes. Cette étude, pionnière dans la région des hautes terres de l'Ouest du Cameroun, vise à identifier les assemblages d'algues en relation avec quelques paramètres physicochimiques de l'eau dans deux sites marécageux.

\section{MATÉRIELS ET MÉTHODES}

\subsection{Sites d'étude}

Les échantillons destinés à l'étude des algues ont été prélevés entre octobre 2003 et février 2004 dans deux sites marécageux de la région de Dschang (Foto et Nkong-ni), département de la Menoua (Ouest-Cameroun). Les prélèvements ont été effectués dans neuf stations dans le site de Foto et six dans le site de Nkong-ni. Cette zone est située à environ 1400 mètres d'altitude à $5^{\circ} 25^{\prime}-5^{\circ} 30^{\prime}$ de latitude Nord et à $10^{\circ} 00^{\prime}-10^{\circ} 20^{\prime}$ de longitude Est (Figure 1). Elle connaît un climat équatorial fortement influencé par le relief dit de type camerounien d'altitude. Ce climat comporte une courte saison sèche qui va de novembre à février et une longue saison humide qui s'étale de mars à octobre. D'après les données instrumentales moyennées sur 28 ans de la station météorologique de l'IRAD (Institut de Recherche Agricole pour le Développement) de Dschang, la pluviométrie annuelle est de $1850 \mathrm{~mm}$, la température moyenne annuelle est de $20,1^{\circ} \mathrm{C}$ avec une amplitude thermique faible $\left(2,2^{\circ} \mathrm{C}\right)$.

\subsubsection{Site de Foto}

Le site marécageux de Foto se trouve à la périphérie immédiate de la ville de Dschang, non loin du campus universitaire. C'est un marécage d'environ 0,3 hectare de superficie, limité de part et d'autre par des pentes abruptes à l'exception de la partie Est où le relief est plat. Il est alimenté par des précipitations, des eaux de ruissellement et des eaux souterraines. L'eau s'écoule par un exutoire situé dans la partie Est du marécage. Le niveau d'eau au moment des prélèvements a fluctué entre 7,92 et $10,93 \mathrm{~cm}$. Le marécage est colonisé par de nombreuses macrophytes dont les plus représentées sont Panicum sp. ${ }_{1}$, Panicum. sp. ${ }_{2}$, Pycreus lanceolatus, Cyperus difformis, Brachiaria sp., Cyclosorus striatus, Floscopa africana (FONKOU et al., 2005).

\subsubsection{Site de Nkong-ni}

Il est situé à environ $12 \mathrm{~km}$ au nord de la ville de Dschang sur la route départementale reliant cette ville à Bafoussam. Il se trouve à cheval entre les villages Bafou et Baleveng. Sa superficie avoisine les dix hectares. Ce marécage est alimenté par la rivière Ndoubou, par les eaux de pluies, les eaux de ruissellement et les sources souterraines. Le niveau d'eau au moment des 


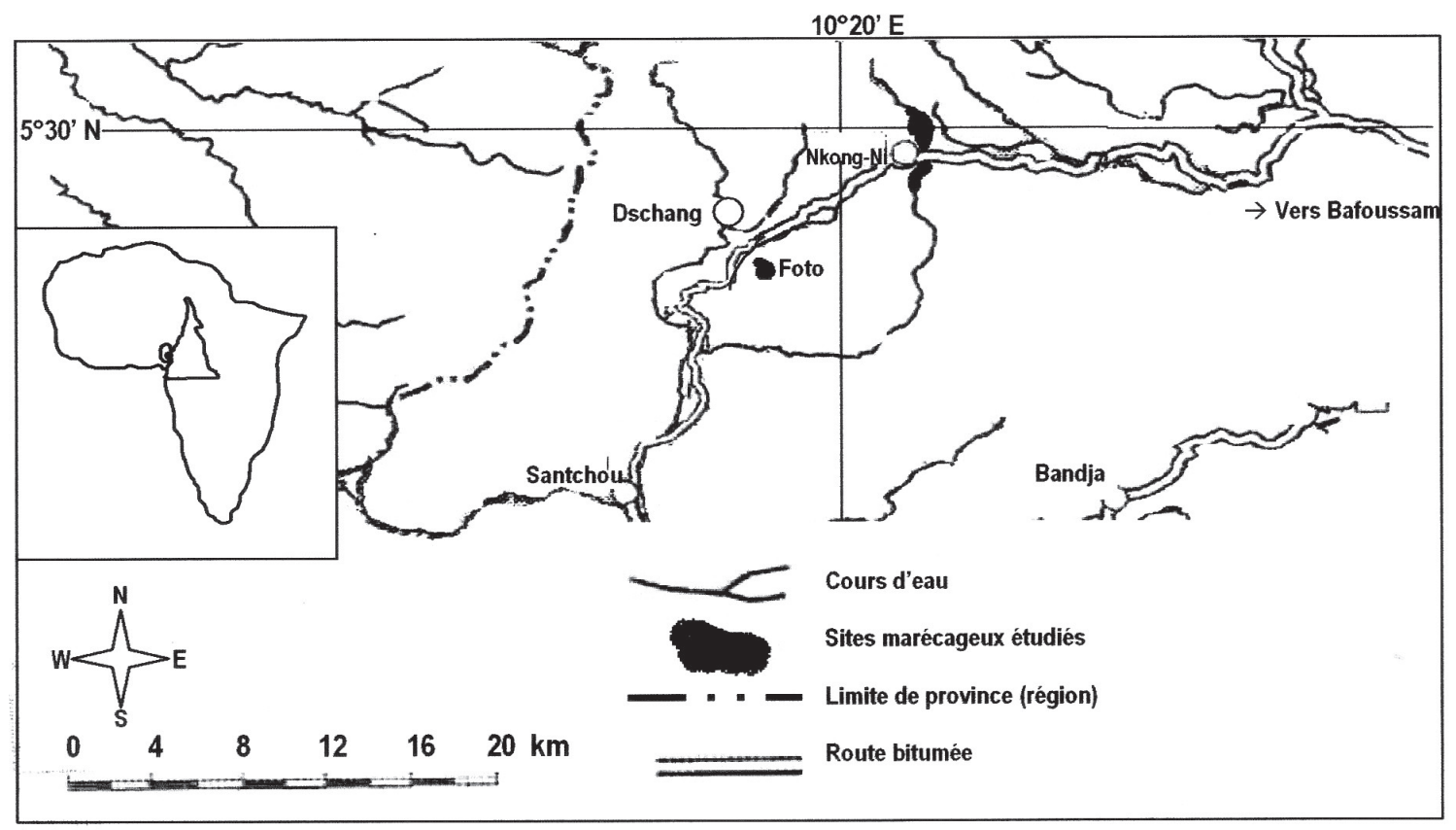

Figure 1. Situation des sites marécageux étudiés dans les hautes terres de l'Ouest du Cameroun. Location of the studied wetland sites in the western highlands of Cameroon.

prélèvements a atteint $90-150 \mathrm{~cm}$ en certains points. Ce site, autrefois occupé par des raphiales, est aujourd'hui colonisé par de nombreuses macrophytes parmi lesquelles les plus importantes sont Rhynchospora corymbosa, Echinochloa cruspavonis, Leersia hexandra, Floscopa africana, Panicum sp. Fuirena umbellata, Aspilia angustifolia, Eleocharis acutangula, Schoenoplectus articulatus (FONKOU et al., 2005).

\section{2 Échantillonnage et conservation des échantillons}

\subsubsection{Sur le terrain}

Les échantillons étudiés ont été prélevés à l'entrée (I) des marécages, au milieu $(\mathrm{M})$ et à la sortie $(\mathrm{O})$ dans le but de détecter les variations spatiales de la flore algale et des paramètres physico-chimiques de l'eau. Au total, 15 échantillons ont été prélevés dont neuf à Foto et six à Nkong-ni. Dans le site de Foto, trois échantillons notés FI1, FI2 et FI3 ("Foto inlet » 1, 2,3) ont été prélevés à l'entrée du marécage suivant un transect Nord-Sud (NS). Ces points de prélèvements correspondent aux débouchés des eaux de ruissellement dans le marécage. Trois échantillons notés FM1, FM2, FM3 ("Foto middle 1, 2,3) puis trois autres notés FO1, FO2 et FO3 ("Foto outlet » $1,2,3)$ ont été prélevés respectivement au milieu et à la sortie du marécage en suivant, comme à l'entrée, une orientation NS. A Nkong-ni les échantillons ont aussi été prélevés le long d'un transect NS. Le niveau d'eau étant relativement important comparé à Foto, nous avons prélevé systématiquement dans chaque point un échantillon du benthos et un échantillon de la colonne d'eau. Les échantillons du plancton prélevés à l'entrée, au milieu et à la sortie du marécage sont notés respectivement NI1, NM1 et NO1. Les échantillons du benthos correspondant sont notés NI2, NM2 et NO2.

Les échantillons destinés à l'étude des algues ont été prélevés par grattage sur différents types de substrats (rochers, objets immergés) et par expression de macrophytes immergées. Ces échantillons ont été recueillis dans des piluliers de $40 \mathrm{~mL}$, puis subdivisés en deux sous-échantillons notés A et $\mathrm{B}$. Dans le lot $\mathrm{A}, 10$ à 15 gouttes de formol dilué à $10 \%$ ont été ajoutées à l'échantillon. Ce prétraitement au formol empêche la dégradation de la matière organique et permet ainsi la fixation des algues. Le lot $\mathrm{B}$ a été conservé sans traitement. Environ deux litres d'eau ont été collectés en certains points de prélèvement et séparés aussi en deux lots notés A1 et B1. Le lot $\mathrm{A} 1$ a été traité au formol dilué à $10 \%$ dans les proportions 3/4 d'échantillon pour $1 / 4$ de formol. Le lot B1 n'a pas été traité. Ce type d'échantillonnage permet de récolter les organismes qui vivent dans l'eau libre en se maintenant par des mécanismes actifs ou passifs. Cette méthode a l'avantage par rapport au filet à plancton de permettre de récolter toutes les catégories de taille d'algues.

\subsubsection{Au laboratoire}

Une à deux gouttes de chaque échantillon non traité (B et B1) ont été montées entre lame et lamelle et observées immédiatement au microscope optique, le but étant d'observer les organismes encore vivants dans leur déplacement et de 
noter certains détails qui pourraient disparaître par le fait de la fixation (couleur pour l'ensemble des espèces et mouvements des flagelles pour les espèces qui se déplacent de façon active).

Chaque échantillon du lot $\mathrm{A} 1$ a été transféré dans un bêcher et laissé au repos pendant 24 heures au minimum pour permettre la sédimentation des algues. L'eau a été ensuite enlevée au-dessus du résidu algal par siphonage. La solution résiduelle concentrée en algues a été conservée dans un pilulier.

Quelques gouttes de chaque échantillon ont été montées entre lame et lamelle et observées à l'aide d'un microscope optique de marque Olympus, modèle $\mathrm{BH}-2$, équipé d'une lentille Nomarski. Pour chaque échantillon, au moins trois lames ont été préparées afin de s'assurer de la reproductibilité des lames. Les comptages ont été faits suivant des transects verticaux choisis au hasard sur la lame jusqu'à obtention de 200 individus au moins. Dans les échantillons très riches en algues, ou dominés par un petit nombre d'espèces, les comptages ont concerné un grand nombre d'individus. Par exemple, dans l'échantillon NO2, 2064 individus ont été comptés dans le but de mettre en évidence les espèces rares. Les identifications ont été faites en utilisant les clés publiées dans les documents de référence (BOURRELLY, 1976; BOURELLY et MANGUIN, 1952; COMPÈRE, 1974, 1976, 1977; COUTÉ et ROUSSELIN, 1975; GASSE, 1980; 1986; ILTIS, 1980; KRAMMER et LANGE-BERTALOT, 1986; 1988; 1991). Le pourcentage de chaque taxon dans l'échantillon a été calculé en fonction du nombre total d'individus comptés.

\section{3 Analyse des paramètres physico-chimiques des eaux}

Dans chaque point de prélèvement, la conductivité, les solides totaux dissous (STD), le $\mathrm{pH}$ et la température ont été mesurés. La conductivité $\left(\mu \mathrm{S} \bullet \mathrm{cm}^{-1}\right)$, les STD $\left(\mathrm{mg}^{\bullet} \mathrm{L}^{-1}\right)$ et la température $\left({ }^{\circ} \mathrm{C}\right)$ ont été mesurées sur le terrain à l'aide d'un STD /Conductimètre de marque Hach. Le $\mathrm{pH}$ des échantillons a été mesuré à l'aide d'un pH-mètre de marque Suntex TS2. La conductivité électrique permet d'évaluer le degré de minéralisation de l'eau, tandis que les STD indiquent la teneur en ions organiques et inorganiques en solution.

\section{4 Analyses statistiques}

Les données floristiques ont été soumises à une Analyse Factorielle des Correspondances (AFC). Le tableau de contingence soumis à cette analyse comprenait les individus (échantillons) en colonnes $\left(\mathrm{x}_{\mathrm{n}}\right)$ et les variables (espèces) en lignes $\left(\mathrm{y}_{\mathrm{p}}\right)$. Chaque case du tableau $\left(\mathrm{x}_{\mathrm{i}} \mathrm{y}_{\mathrm{i}}\right)$ correspond à l'effectif de l'espèce $\mathrm{y}_{\mathrm{i}}$ dans l'échantillon $\mathrm{x}_{\mathrm{i}}$. L'AFC est une méthode d'ordination qui va permettre de détecter dans les données à analyser les grands axes de variations en se basant sur les effectifs des différentes espèces dans les différents échantillons. L'analyse va affecter à ces échantillons et espèces des coordonnées dans un espace vectoriel de dimension $\mathrm{n}-1, \mathrm{n}$ étant le nombre d'espèces. Elle va extraire successivement des axes orthogonaux et donc non corrélés; les axes les plus intéressants sont ceux qui correspondent aux allongements maximum du nuage de points. Les résultats se présentent généralement sous forme de plans factoriels où chaque axe est considéré comme un gradient hypothétique qui est ensuite interprété en se basant sur les valeurs des paramètres environnementaux des échantillons et l'écologie des espèces ayant une forte contribution sur l'axe.

Les échantillons d'eau libre et ceux prélevés par grattage ont été réunis dans le même tableau de données, dans le but vérifier si l'habitat ou les conditions physico-chimiques dans chaque type d'habitat étaient des facteurs importants dans la distribution spatiale des microphytes.

\section{RÉSULTATS ET DISCUSSION}

\subsection{Caractéristiques physico-chimiques des eaux des marécages}

Le tableau 1 montre les statistiques simples des résultats des analyses physico-chimiques effectuées.

Dans le site de Foto, la conductivité électrique varie entre 43,7 à $146,9 \mu \mathrm{S}^{\bullet} \mathrm{cm}^{-1}$ et les STD entre 21,8 et $73,60 \mathrm{mg}^{\bullet} \mathrm{L}^{-1}$. Les intervalles de variations de ces paramètres sont beaucoup plus faibles à Nkong-ni $\left(35,8\right.$ à $51,5 \mu S^{\bullet} \mathrm{cm}^{-1}$ et 17,9 à $25,7 \mathrm{mg} \mathrm{L}^{-1}$ ), indiquant une plus grande variabilité des milieux aquatiques à Foto comparés à Nkong-ni. À Foto, la valeur la plus faible de conductivité $\left(44 \mu \mathrm{S} \bullet \mathrm{cm}^{-1}\right)$ est obtenue au point FI1 (Figure 2A) situé à l'entrée du marécage alors que les valeurs les plus élevées $\left(>100 \mu \mathrm{S} \bullet \mathrm{cm}^{-1}\right)$ sont enregistrées au milieu (FM1) ou à la sortie du marécage (FO1 et FO3). Ces fortes valeurs de conductivité coïncident avec les valeurs les plus élevées de STD $\left(53,0\right.$ à $\left.73,6 \mathrm{mg}^{\bullet} \mathrm{L}^{-1}\right)$. Ces paramètres dans les échantillons prélevés à Nkong-ni étaient faibles comparés à ceux de Foto (Figure 2B); les valeurs moyennes de ces paramètres étaient donc corrélativement plus élevées à Foto, montrant que les eaux de ce site sont plus chargées en ions. Néanmoins, ces valeurs moyennes sont restées faibles par rapport à celles obtenues dans d'autres sites dans les hautes terres de l'Ouest-Cameroun (FONKOU et al., 2005; NGUETSOP et al., 2007), dans le sud du Cameroun (KEMKA et al., 2006), et dans certains sites sahéliens/sahariens où des valeurs de conductivité supérieures à $400 \mu S^{\bullet} \mathrm{cm}^{-1}$ ont été enregistrées (GASSE, 2002).

Le pH des eaux au site de Foto a varié entre 6,07 et 6,58, les valeurs les plus faibles étant observées au niveau de la station FI1 à l'entrée du marécage et au niveau de la station FO2 à la sortie du marécage. Les valeurs les plus élevées ont été enregistrées à FM1 et FM3 au milieu du marécage (Figure 2C). Le pH des 
Tableau 1. Statistiques simplifiées des paramètres physico-chimiques mesurés dans les marécages.

Table 1. Simplified statistics of the physicochemical parameters measured in the wetlands.

\begin{tabular}{ccccccc}
\hline \multirow{2}{*}{ Sites } & Variables & Minimum & Maximum & Moyenne & Écart-type & Mediane \\
\hline \multirow{4}{*}{ Foto } & Conductivité $\left(\mu \mathrm{S}^{*} \mathrm{~cm}^{-1}\right)$ & 43,75 & 146,88 & 89,54 & 32,28 & 82,60 \\
& $\mathrm{STD}\left(\mathrm{mg} \bullet \mathrm{L}^{-1}\right)$ & 21,79 & 73,60 & 44,73 & 16,30 & 41,20 \\
& $\mathrm{~T}\left({ }^{\circ} \mathrm{C}\right)$ & 23,23 & 25,83 & 24,14 & 0,83 & 24,05 \\
& $\mathrm{pH}$ & 6,07 & 6,58 & 6,33 & 0,18 & 6,33 \\
& Profondeur $(\mathrm{cm})$ & 7,92 & 10.93 & 8,85 & 1,80 & 7,92 \\
\hline \multirow{3}{*}{ Nkong-ni } & $\mathrm{Conductivité}\left(\mu \mathrm{S} \bullet \mathrm{cm}^{-1}\right)$ & 35,80 & 51,50 & 41,10 & 9,00 & 36,00 \\
& $\mathrm{STD}\left(\mathrm{mg} \cdot \mathrm{L}^{-1}\right)$ & 17,90 & 25,70 & 20,53 & 4,47 & 18,00 \\
& $\mathrm{~T}\left({ }^{\circ} \mathrm{C}\right)$ & 22,40 & 22,60 & 22,53 & 0,12 & 22,60 \\
& $\mathrm{pH}$ & 6,64 & 6,81 & 6,70 & 0,09 & 6,66 \\
& Profondeur $(\mathrm{cm})$ & 90,00 & 150,00 & 118,33 & 30,14 & 115,00 \\
\hline
\end{tabular}
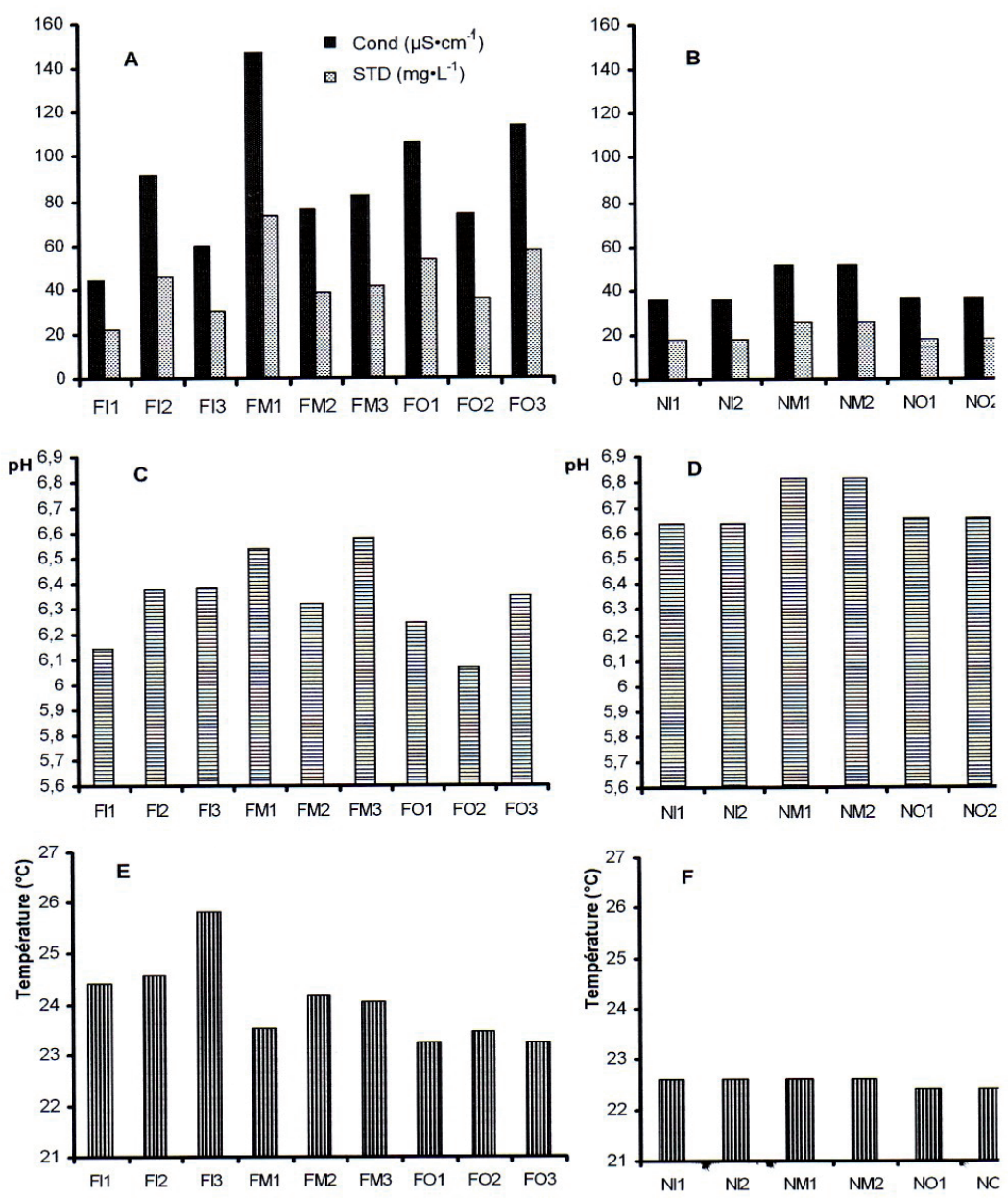

Figure 2. Caractéristiques physico-chimiques de l'eau aux points de prélèvement : variations des valeurs de STD $\left(\mathrm{mg}^{\bullet} \mathrm{L}^{-1}\right)$ et de la conductivité $\left(\mu \mathrm{S}^{\bullet} \mathrm{cm}^{-1}\right)$ dans les sites de Foto (A) et Nkong-ni (B); variations des valeurs de $\mathrm{pH}$ à Foto $(\mathrm{C})$ et à Nkong-ni $(\mathrm{D})$; variations des valeurs de température $\left({ }^{\circ} \mathrm{C}\right)$ de l'eau à Foto $(\mathrm{E})$ et Nkong-ni (F).

Physico-chemical characteristics of water at the sampling locations: variations of the TDS and the conductivity in the Foto $(A)$ and the Nkong-Ni (B) sites; variations of the pH at the Foto $(C)$ and the Nkong-Ni (D); variations of the temperature at Foto (E) and Nkong-Ni (F). 
eaux a été globalement plus élevé à Nkong-ni, mais la même tendance dans l'évolution spatiale a été observée (Figure 2D). Ainsi, dans les deux sites, les eaux ont été légèrement acides, mais l'écart-type est passé de 0,09 à Nkong-ni à 0,18 à Foto. Ces différences seraient liées à l'origine des deux marécages, et à leur composition macrophytique. En effet, l'alimentation en eau ne se fait pas de la même manière dans les deux marécages. De plus, le degré d'adaptation des macrophytes aux conditions d'anoxie régnant dans les substrats pourrait avoir une influence sur le degré d'oxygénation du substrat, et donc du potentiel redox. Les eaux à l'entrée et à la sortie des marécages étant plus en contact avec l'air atmosphérique que l'eau infiltrée, il serait évident que les processus d'oxydation ne se déroulent pas avec la même ampleur.

La température des eaux au moment des prélèvements a varié entre 22,23 et $25,83^{\circ} \mathrm{C}$ à Foto et entre 22,40 et $22,60^{\circ} \mathrm{C}$ à Nkong-ni. A priori on pourrait penser, d'après les figures $2 \mathrm{E}$ et $2 \mathrm{~F}$ et les écart-types obtenus dans les deux sites, à une plus grande variabilité spatiale de la température à Foto mais les données de ce paramètre doivent être interprétées avec beaucoup de prudence. En effet, dans les latitudes tropicales et subtropicales, la température présente une forte variabilité à l'échelle de la journée (MISKANE, 1997).

Le niveau des eaux est resté faible; toutefois il a été relativement plus élevé à Nkong-ni (entre 90 et $150 \mathrm{~cm}$ ) qu’à Foto (entre 7,92 et 10,83 cm). C'est le seul paramètre montrant un écart-type plus élevé à Nkong-ni $(30,14)$ comparé à Foto $(1,80)$.

\subsection{Analyse qualitative de la flore}

Les observations au microscope optique ont permis d'identifier dans les 15 échantillons 30 espèces réparties dans quatre embranchements, cinq classes, dix familles et 21 genres (Tableau 2). La classe des Zygophyceae comprend le plus grand nombre de taxons; Spirogyra spp., Cosmarium spp., Closterium spp., Euastrum dubium var. latum, Mougeotia sp. et Pleurotaenium sp. Elle est suivie par la classe des Bacillariophyceae (Diatomées) qui compte sept espèces appartenant aux genres Navicula, Pinnularia, Cymbella, Gomphonema et Tabellaria puis les Euglenophyceae avec quatre espèces appartenant aux genres Euglena, Phacus et Trachelomonas. Les Cyanophyceae sont représentées par trois espèces appartenant aux genres Oscillatoria et Lyngbya. Les Ulotrichophyceae sont les moins représentées avec seulement deux espèces, Uronema sp. et Oedogonium sp. Le tableau 3 montre la répartition des différentes espèces rencontrées dans les échantillons étudiés.

La figure 3 représentant la variation du nombre d'espèces dans les échantillons montre qu'à Nkong-ni le nombre d'espèces a été faible à l'entrée et est devenu plus important à la sortie du marécage, alors qu'à Foto c'est plutôt une tendance inverse qui a été observée. À Nkong-ni, ce nombre a été plus faible dans la colonne d'eau (NI1, NM1 et NO1) que dans les échantillons des habitats benthiques ou épiphytes (NI2, NM2, NO2). À Foto, par contre, le nombre d'espèces a été invariablement élevé ou faible dans les deux catégories d'échantillons. Cela pourrait s'expliquer par le fait que la profondeur étant faible dans ce deuxième site, les conditions écologiques ont été proches dans les deux types d'habitats.

\subsection{Analyse quantitative de la flore}

Cette analyse s'appuie fortement sur les groupes d'échantillons et les assemblages d'algues associés définis par l'AFC. Cette approche globale permet de visionner l'ensemble des échantillons et des espèces dans un nuage de points multidimensionnel. L"interprétation des groupes d'échantillons et non pas d'échantillons isolés permet de palier,

Tableau 2. Espèces d'algues et groupes taxonomiques identifiés dans les échantillons étudiés.

Table 2. $\quad$ Algae species and taxa groups identified in the samples studied.

\begin{tabular}{ccccc}
\hline Embranchements & Classes & Familles & Nombre de genres & Nombre d'espèces \\
\hline \multirow{2}{*}{ Chysophyta } & Bacillariophyceae & Naviculaceae & 4 & 4 \\
& & Fragilariaceae & 2 & 2 \\
& Eunotiaceae & 1 & 1 \\
Chlorophyta & Ulotrichophyceae & Oedogoniaceae & 1 & 1 \\
& & Ulotricaceae & 1 & 1 \\
& Zygophyceae & Zygnemataceae & 2 & 5 \\
& Desmidiaceae & 2 & 8 \\
Euglenophyta & Mesotaeniaceae & 1 & 4 \\
& Euglenophyceae & Euglenaceae & 3 & 3 \\
\hline
\end{tabular}


Tableau 3. Distribution des taxons d'algues rencontrés dans les échantillons étudiés. La croix (x) dans une case indique la présence de l'espèce en ligne dans l'échantillon en colonne.

Table 3. Distribution of identified algae taxa in studied samples. The cross $(x)$ in a given space indicates the presence of the species (row) in a given sample (column).

\begin{tabular}{|c|c|c|c|c|c|c|c|c|c|c|c|c|c|c|c|}
\hline \multirow{2}{*}{ Espèces } & \multicolumn{15}{|c|}{ Échantillons } \\
\hline & FI1 & FI2 & FI3 & FM1 & FM2 & FM3 & FO1 & FO2 & FO3 & NI1 & NI2 & NM1 & NM2 & NO1 & $\mathrm{NO} 2$ \\
\hline Closterium leibleinii & & & & & & & & & & $\mathrm{x}$ & $\mathrm{x}$ & $\mathrm{x}$ & $\mathrm{x}$ & & $\mathrm{x}$ \\
\hline Netrium sp. & $\mathrm{x}$ & $\mathrm{x}$ & $\mathrm{x}$ & $\mathrm{x}$ & & & & $\mathrm{x}$ & & $\mathrm{x}$ & $\mathrm{x}$ & & & & $\mathrm{x}$ \\
\hline Closterium parvulum & $\mathrm{x}$ & $\mathrm{x}$ & $\mathrm{x}$ & & & $\mathrm{x}$ & & & $\mathrm{x}$ & & & & & $\mathrm{x}$ & $\mathrm{x}$ \\
\hline Cosmarium cf scrobiculatum & & & & & & & & & & & & & $\mathrm{x}$ & & $\mathrm{x}$ \\
\hline Cosmarium turpinii & & & & & & & & & & & & & & $\mathrm{x}$ & $\mathrm{x}$ \\
\hline Cosmarium difficile & & & & & & & & & & & & $\mathrm{x}$ & & & $\mathrm{x}$ \\
\hline Cosmarium globossum & & & $\mathrm{x}$ & & & & & & & & & $\mathrm{x}$ & & $\mathrm{x}$ & $\mathrm{x}$ \\
\hline Cymbella silesiaca & $\mathrm{x}$ & $\mathrm{x}$ & $\mathrm{x}$ & $\mathrm{x}$ & $\mathrm{x}$ & $\mathrm{x}$ & & & & & $\mathrm{x}$ & $\mathrm{x}$ & $\mathrm{x}$ & $\mathrm{x}$ & $\mathrm{x}$ \\
\hline Euastrum dubium var. latum & & & & & & & & & & & & & & & $\mathrm{x}$ \\
\hline Euglena sp. & $\mathrm{x}$ & & $\mathrm{x}$ & $\mathrm{x}$ & $\mathrm{x}$ & & & & & & & $\mathrm{x}$ & $\mathrm{x}$ & & $\mathrm{x}$ \\
\hline Eunotia incisa & & & & & & & & & & & $\mathrm{x}$ & & & & $\mathrm{x}$ \\
\hline Fragilaria javanica & & & & & & & & & & & $\mathrm{x}$ & $\mathrm{x}$ & $\mathrm{x}$ & $\mathrm{x}$ & $\mathrm{x}$ \\
\hline Gomphonema parvulum & $\mathrm{x}$ & $\mathrm{x}$ & $\mathrm{x}$ & $\mathrm{x}$ & $\mathrm{x}$ & $\mathrm{x}$ & & & & & $\mathrm{x}$ & & $\mathrm{x}$ & $\mathrm{x}$ & $\mathrm{x}$ \\
\hline Lyngbya sp. & & & & & & & & $\mathrm{x}$ & $\mathrm{x}$ & & & $\mathrm{x}$ & $\mathrm{x}$ & & $\mathrm{x}$ \\
\hline Mougeotia sp. & & & & & & & & & & & & & $\mathrm{x}$ & & \\
\hline Navicula sp. & $\mathrm{x}$ & $\mathrm{x}$ & $\mathrm{x}$ & $\mathrm{x}$ & $\mathrm{x}$ & & $\mathrm{x}$ & $\mathrm{x}$ & $\mathrm{x}$ & $\mathrm{x}$ & $\mathrm{x}$ & $\mathrm{x}$ & $\mathrm{x}$ & $\mathrm{x}$ & $\mathrm{x}$ \\
\hline Oedogonium sp. & & & $\mathrm{x}$ & & & $\mathrm{x}$ & $\mathrm{x}$ & $\mathrm{x}$ & $\mathrm{x}$ & & & & & $\mathrm{x}$ & $\mathrm{x}$ \\
\hline Oscillatoria bornetii & & & & & & & & & & & $\mathrm{x}$ & & $\mathrm{x}$ & & \\
\hline Oscillatoria cf chlorine & $\mathrm{x}$ & $\mathrm{x}$ & $\mathrm{x}$ & $\mathrm{x}$ & $\mathrm{x}$ & & $\mathrm{x}$ & $\mathrm{x}$ & & & $\mathrm{x}$ & & $\mathrm{x}$ & $x$ & $\mathrm{x}$ \\
\hline Phacus sp. & $\mathrm{x}$ & $\mathrm{x}$ & $\mathrm{x}$ & $\mathrm{x}$ & & & & & & $\mathrm{x}$ & $\mathrm{x}$ & & & & $\mathrm{x}$ \\
\hline Pinnularia subgibba & $\mathrm{x}$ & $\mathrm{x}$ & $\mathrm{x}$ & $\mathrm{x}$ & & & & & $\mathrm{x}$ & $\mathrm{x}$ & $\mathrm{x}$ & $\mathrm{x}$ & $\mathrm{x}$ & $\mathrm{x}$ & $\mathrm{x}$ \\
\hline Pleurotaenium sp. & & & & & & & & & & & $\mathrm{x}$ & & & & $\mathrm{x}$ \\
\hline Spirogyra variformis & $\mathrm{x}$ & & & & & & $\mathrm{x}$ & & & & & & & & \\
\hline Spirogyra corrugata & & $\mathrm{x}$ & $\mathrm{x}$ & $\mathrm{x}$ & $\mathrm{x}$ & & $\mathrm{x}$ & $\mathrm{x}$ & $\mathrm{x}$ & & & & & & \\
\hline Spirogyra decimina & $\mathrm{x}$ & $\mathrm{x}$ & $\mathrm{x}$ & & & & & $\mathrm{x}$ & & & & & $\mathrm{x}$ & & $\mathrm{x}$ \\
\hline Spirogyra occidentalis & & & & & & & & $\mathrm{x}$ & & & & & & & \\
\hline Tabellaria floculosa & & & & & & & & & & & $\mathrm{x}$ & $\mathrm{x}$ & $\mathrm{x}$ & $\mathrm{x}$ & $\mathrm{x}$ \\
\hline Trachelomonas planctonica & $\mathrm{x}$ & $\mathrm{x}$ & $\mathrm{x}$ & $\mathrm{x}$ & & & & & & & & & & & $\mathrm{x}$ \\
\hline Trachelomonas volvocinopsis & & & & & & & & & & & & & & & $\mathrm{x}$ \\
\hline Uronema sp. & & & & & & & & & & & & & & & $\mathrm{x}$ \\
\hline
\end{tabular}



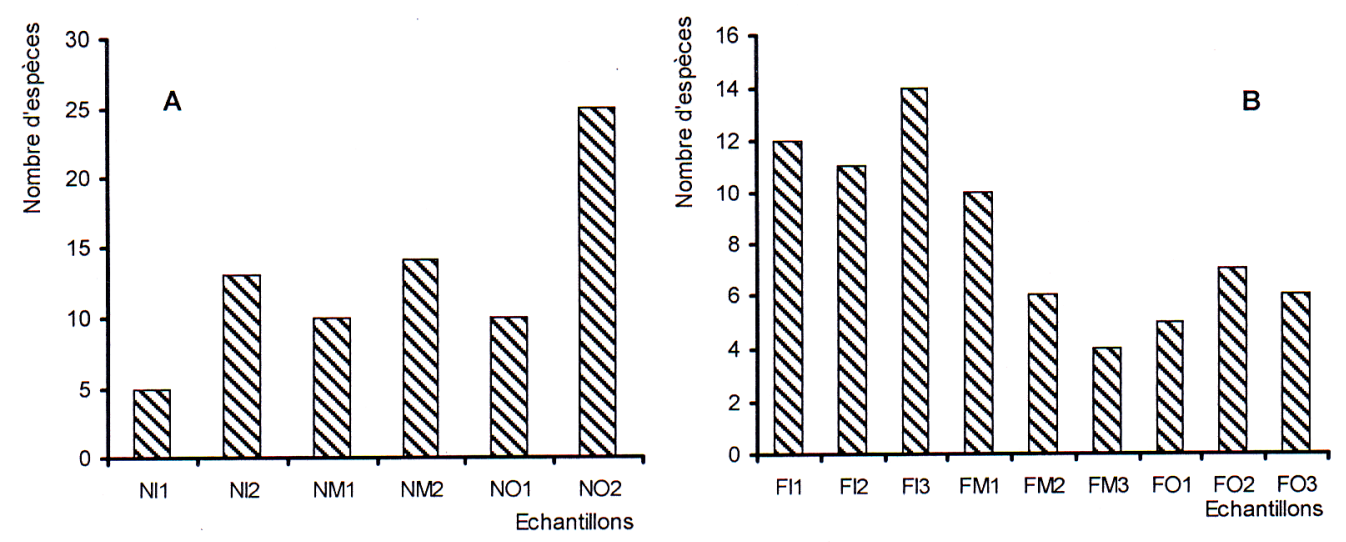

Figure 3. Variation du nombre d'espèces dans les échantillons observées dans les marécages de Foto (A) et Nkong-Ni (B). NI, entrée du marécage de Nkong-Ni; NM, milieu du marécage de Nkong-Ni; NO, sortie du marécage de Nkong-Ni; FI, entrée du marécage de Foto; FM, milieu du marécage de Foto; FO, Sortie du marécage de Foto.

Variation of the number of species in the Foto $(A)$ and Nkong-Ni (B) wetlands. NI, entry of the Nkong-Ni wetland; NM, centre of the Nkong-Ni wetland; NO, outlet of the Nkong-Ni wetland; FI, entry of the Foto wetland; FM, centre of the Foto wetland; FO, outlet of the Foto wetland.

au moins en partie, au manque de répétitions temporelles dans l'échantillonnage qui permettrait de mieux comprendre le comportement des espèces vis-à-vis des paramètres physicochimiques du milieu aquatique.

\subsubsection{Signification écologique des assemblages}

La figure 4 représente le plan 1-2 de l'analyse factorielle des correspondances (AFC). L'axe 1 (33,8 \% d'inertie) oppose le groupe I situé du côté négatif au groupe II situé du côté positif de l'axe. Le groupe I comprend les échantillons FO1, FO2 et FI3, tous prélevés dans le site de Foto à l'entrée (FI3) ou à la sortie du marécage. Les échantillons NI2, NM2 et NO2 qui forment le groupe II proviennent du site de Nkong-ni à l'entrée (NI2), au milieu (NM2) ou à la sortie (NO2) du marécage. Les Chlorophyceae, Spirogyra corrugata (Spi2), S. decimina (Spi3) et $S$. occidentalis (Spi4) sont associées au groupe I alors que le groupe II est caractérisé par Cosmarium cf scrobiculatum, Eunotia incisa, Gomphonema parvulum, Navicula sp. et Tabellaria floculosa.

Les points de prélèvement des échantillons du groupe I sont des points d'eau peu profonds, bien éclairés et à courant relativement faible. Les spirogyres ont été observées dans des eaux de faibles profondeurs colonisées par des macrophytes, sans fixation dans la tranche d'eau ou au niveau des sédiments (TRÉMOLIÈRES, 2004). Dans les points de prélèvement des échantillons du groupe I (Foto), les spirogyres peuvent donc proliférer en adhérant sur la vase ou sur des matériaux immergés; elles forment parfois une enveloppe mucilagineuse autour des tiges de macrophytes immergées. Les échantillons de Nkongni par contre proviennent du grattage des dalles en béton immergées (NI2 et NO2) ou de débris organiques immergés
(NM2), et le courant est relativement plus élevé comparé à Foto. On pourrait donc penser en première approximation que l'axe constitue un gradient de courant mais cette hypothèse reste difficile à confirmer en l'absence de données de courant. Il est possible que l'axe différencie plutôt les types d'habitats car tous les échantillons du groupe II ont été prélevés par grattage à Nkong-ni alors que ceux du groupe I proviennent de points d'eau de faibles profondeurs à Foto. Mais le fait que les échantillons prélevés par grattage à Foto ne figurent pas dans le groupe I montre que l'habitat ne serait pas le seul facteur qui conditionne la position des taxons le long de l'axe I. Lorsqu'on considère l'écologie des espèces qui caractérisent l'axe 1 , on constate que l'axe oppose les espèces indicatrices de fortes pollutions organiques aux espèces oligotrophes, c'est-à-dire qui prolifèrent dans des eaux pauvres en matières organiques. En effet, les spirogyres ont été trouvées en abondance dans les eaux à fortes pollutions organiques (BONNARD et al., 2003; FOLEFACK, 1989; NGUETSOP, 1990; USEPA, 2003). Les taxons Eunotia spp., Gomphonema spp. et Navicula spp. par contre sont réputés oligotrophes (HUSTEDT, 1930; KRAMMER et LANGE-BERTALOT, 1986, 1991); ils peuvent vivre comme épiphytes sur les macrophytes ou en épilithique sur des substrats relativement durs. Les Tabellaria spp. sont considérées comme tychoplanctoniques (GASSE, 1986); elles peuvent donc connaître des stades fixés au cours de leur cycle de vie. Cette dernière hypothèse est renforcée lorsque l'on considère les données de STD et de conductivité des échantillons ayant une forte contribution sur l'axe; les fortes valeurs de ces paramètres suggèrent généralement de fortes pollutions organiques ou minérales (HACH, 1997). Globalement, les échantillons du groupe I présentent des valeurs de STD et de conductivité relativement élevées alors que les échantillons du 


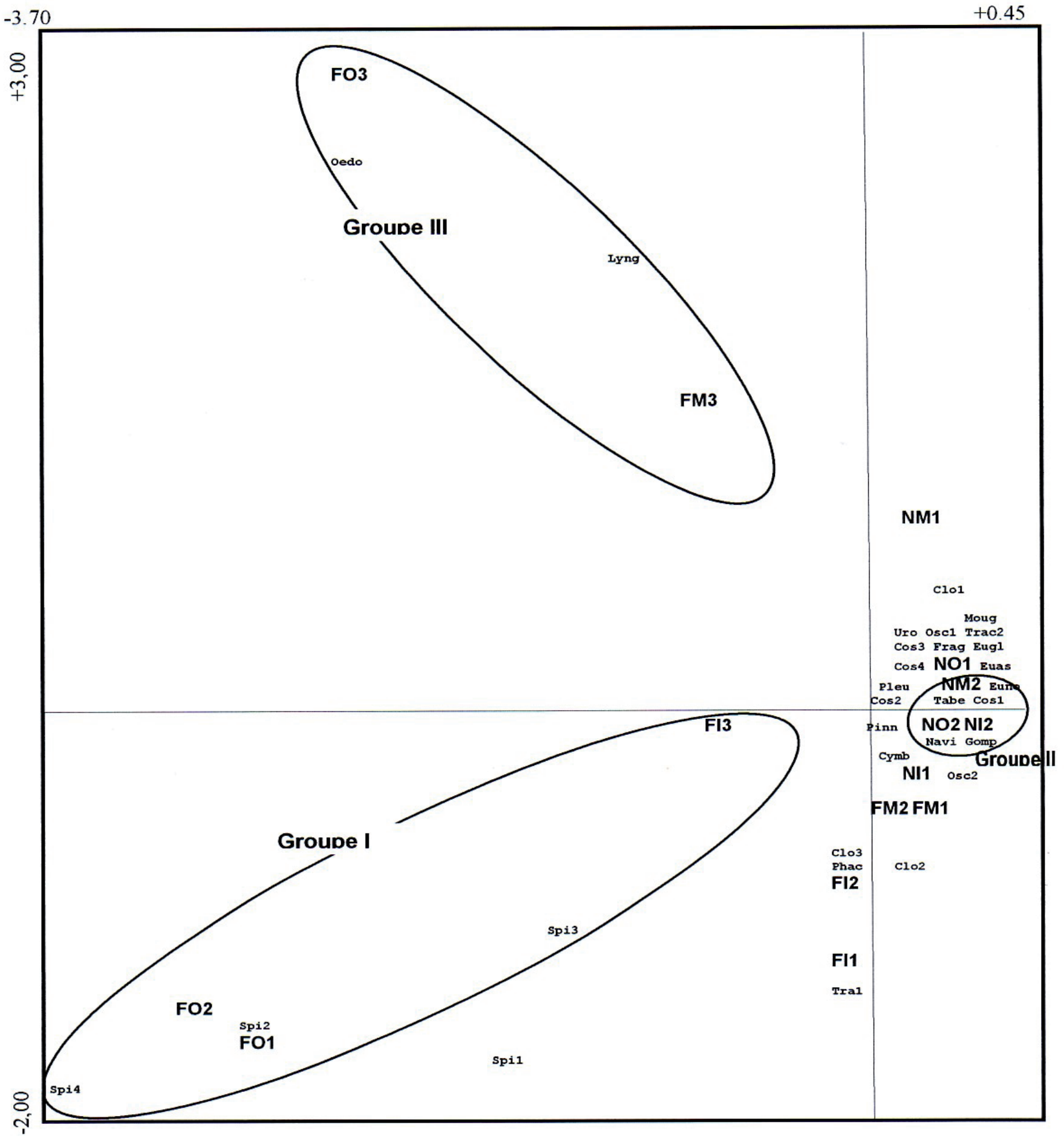

Figure 4. Représentations des groupes (I, II et III), des échantillons (en gras) et des espèces dans le plan factoriel 1-2 de l'AFC. (Spi1, Spiropyra variformis; Spi2, S. corrugata; Sp3, S. decimina; Sp4, S. occidentalis; Oedo, Oedogonium sp.; Lyng, Lyngbya sp.; Osc1, Oscillatoria bornetii; Osc2, Oscillatoria cf chlorine; Clo1, Closterium leibleinii; Clo3, C. parvulum; Clo2, Netrium sp.; Cos1, Cosmarium cf scrobiculatum; Cos2, C. turpinii; Cos3, C. difficile; Cos4, C. globossum; Euas, Euastrum dubium var. latum; Pleuro, Pleurotaenium sp; Uro, Uronema sp.; Moug, Mougeotia sp.; Eugl, Euglena sp.; Phac, Phacus sp.; Trac1, Trachelomonas planctonica; Trac2, T. volvocinopsis; Frag, Fragilaria javanica; Tabe, Tabellaria floculosa; Euno, Eunotia incisa; Cymb, Cymbella silesiaca; Navi, Navicula sp.; Gomp, Gomphonema parvulum; Pinn, Pinnularia subgibba).

Representations of the groups (I, II and III) of the samples (in bold) and the species in the biplot 1-2 of the AFC. (Spil, Spiropyra variformis; Spi2, S. corrugata; Sp3, S. decimina; Sp4, S. occidentalis; Oedo, Oedogonium sp.; Lyng, Lyngbya sp.; Osc1, Oscillatoria bornetii; Osc2, Oscillatoria cf chlorine; Clo1, Closterium leibleinii; Clo3, C. parvulum; Clo2, Netrium sp.; Cos1, Cosmarium cf scrobiculatum; Cos2, C. turpinii; Cos3, C. difficile; Cos4, C. globossum; Euas, Euastrum dubium var. latum; Pleuro, Pleurotaenium sp.; Uro, Uronema sp.; Moug, Mougeotia sp.; Eugl, Euglena sp.; Phac, Phacus sp.; Trac1, Trachelomonas planctonica; Trac2, T. volvocinopsis; Frag, Fragilaria javanica; Tabe, Tabellaria floculosa; Euno, Eunotia incisa; Cymb, Cymbella silesiaca; Navi, Navicula sp.; Gomp, Gomphonema parvulum; Pinn, Pinnularia subgibba). 
groupe II ont des valeurs plutôt faibles. Finalement, les analyses physico-chimiques et l'écologie des espèces telles que montrées par la littérature (voir par exemple, ILTIS, 1980; KEMKA et al., 2004, 2006) suggèrent fortement que l'axe 1 est un axe de pollution organique.

Cette différence dans la composition physico-chimique des eaux des deux sites, et donc de la flore algale, pourrait être fonction de leur composition en macrophytes. En effet, les espèces de macrophytes les plus importantes communes aux deux sites étaient Floscopa africana, et Panicum sp.1. Les autres espèces importantes à Foto ne sont apparues qu'avec des pourcentages faibles à Nkong-ni. Inversement, les autres espèces montrant de forts pourcentages à Nkong-ni n'ont été que faiblement représentées à Foto. Par ailleurs, les macrophytes, par leur structure morphologique (taille, type d'appareil foliaire), pourraient influer sur les paramètres comme l'éclairement incident et l'oxygénation de la colonne d'eau. Elles constitueraient aussi des structures de stockage de la matière organique et de nutriments qui seraient ensuite remis dans l'eau ou dans les sédiments pendant la période de sénescence et de dégradation de la matière organiques (TESTARD, 1995). En conditions expérimentales, il a été démontré que les macrophytes ou leurs extraits sont capables de modifier la composition du phytoplancton (IWONA, 1995). Il est donc fort probable que les différences observées dans la distribution qualitative et quantitative des macrophytes dans l'espace aient influencé, au moins partiellement, la variabilité spatiale des conditions physico-chimiques et corrélativement la distribution des taxons d'algues dans les marécages.

L'axe 2 (19,6 \% d'inertie) individualise du côté positif le groupe III comprenant les échantillons FO3 et FM3 prélevés à Foto. Ils sont associés aux espèces Oedogonium sp. et Lyngbya sp. Ce groupe est difficile à interpréter en matière de gradient environnemental car aucun groupe n'est individualisé du côté négatif de l'axe. Les points de prélèvement de FO3 et FM3 étant caractérisés par des courants relativement forts, le courant pourrait donc être le facteur discriminant de l'axe. Cependant, en l'absence de mesures absolues de courant, il n'est pas possible de conclure que ce paramètre est le facteur prépondérant sur l'axe 2. L'ordination des échantillons et des espèces le long de l'axe peut être également liée à un paramètre environnemental qui n'a pas été pris en compte dans le cadre de cette étude, par exemple l'azote minéral $\left(\mathrm{NO}_{3}^{-}, \mathrm{NH}_{4}^{+}, \mathrm{NO}_{2}^{-}\right)$qui représente l'un des nutriments quantitativement importants pour les algues (DAUTA et FEUILLADE, 1995), les phosphates, l'oxygène dissous etc. Le faible pourcentage d'inertie de l'axe indique que très peu d'échantillons et d'espèces sont pris en compte dans la construction de l'axe.

\subsubsection{Variabilité spatiale et temporelle des assemblages et des paramètres physico-chimiques}

L'AFC individualise trois espèces de spirogyres qui suggèrent de fortes pollutions organiques dans les points de prélèvement. Cela est renforcé par les valeurs élevées de STD et de conductivité qui peuvent aussi être considérées comme des indices de pollution organique. Cependant, dans d'autres sites où la conductivité et les STD sont relativement élevées, suggérant une pollution relativement élevée, ces spirogyres sont absentes. Cela montre que, même si la pollution est le principal facteur induisant leur prolifération, cette pollution devrait probablement être associée à d'autres facteurs. On peut penser par exemple à l'absence ou à un éloignement relatif des macrophytes par rapport à leur site de développement et corrélativement une production qualitative et quantitative différente de matière organique particulaire ou dissoute.

Certains taxons réputés indicateurs de fortes pollutions organiques dans les écosystèmes limniques tels que Phacus spp., Euglena spp. et Tachelomonas spp. (voir par exemple AMOUGOU et al., 1999; FOLEFACK, 1989) sont observés dans la présente étude, parfois dans des sites où les STD et la conductivité sont faibles (FI1, FI2, NO2 et NM1). Euglena gracilis et Phacus helicoïdes atteignent des valeurs de 24 et $58 \%$ respectivement dans le plancton du lac municipal de Yaoundé qui est hypereutrophe; Trachelomonas volvocinopsis rencontrée dans le marécage de Nkong-Ni (NO2) atteint $16 \%$ dans le même lac (KEMKA et al., 2004). Il est possible que la présence de ces taxons soit liée à un paramètre physico-chimique qui n'a pas été mesuré dans le cadre de cette étude. Dans tous les cas, d'après les données disponibles, ce paramètre influencerait peu la distribution spatiale de ces taxons d'algues car ces espèces ne sont pas individualisées dans les groupes des deux premiers axes factoriels de l'AFC (58,4\% d'inertie). Ces espèces apparaissent dans les échantillons étudiés de Foto et Nkong-ni en mélange avec des taxons oligotrophes et benthiques/épiphytes tels que Pinnularia subgibba, Navicula sp. Cymbella silesiaca, et Gomphonema parvulum. Ainsi, même si la variabilité temporelle n'a pas été étudiée dans ce travail, on peut supposer que les espèces eutrophes, en admettant qu'elles n'aient pas été transportées, se sont développées à une période de l'année où les eaux étaient fortement chargées en matière organique et que les espèces oligotrohes seraient devenues prépondérantes lorsque les eaux étaient moins chargées. Cela témoignerait de la variabilité des conditions physico-chimiques de l'eau à l'échelle de plusieurs semaines à plusieurs mois. 
Cette analyse met donc en évidence une variabilité spatiotemporelle des conditions limnologiques qui est reflétée aussi dans les assemblages d'algues. Le faible taux de pollution organique (déduit des valeurs faibles de conductivité et de STD ou de l'écologie des assemblages d'algues) dans les sites à courant d'eau relativement fort peut s'expliquer par une autoépuration rapide; ici, les matières polluantes sont rapidement exportées hors de l'écosystème. Par contre, dans les milieux d'eaux stagnantes ou à courant faible, la variabilité spatiale de la pollution peut s'expliquer par la position du site par rapport à l'arrivée des polluants. Dans ce cas, l'homogénéisation des eaux ne se fait pas complètement.

\section{CONCLUSIONS}

L'étude des assemblages d'algues dans les échantillons d'eau provenant des deux sites marécageux de l'Ouest-Cameroun montre que la flore algale est assez riche. La répartition des espèces dans l'espace semble être contrôlée principalement par la pollution organique qui semble elle-même liée à la distribution spatiale des macrophytes. Le mélange des espèces de préférences écologiques différentes dans le même échantillon, c'est-àdire la contradiction entre l'écologie d'après les données de la littérature et celle déduite des paramètres chimiques mesurés, pourrait être attribué à la variabilité à courte échelle de temps des conditions physico-chimiques car les sites se trouvent en environnements contrastés. À cette variabilité temporelle se superpose une variabilité spatiale qui peut être expliquée par la direction privilégiée des polluants organiques ou par la répartition dans l'espace des macrophytes.

Il est cependant nécessaire d'agrandir la base de données, d'échantillonner au cours de plusieurs mois de l'année, et d'identifier toutes les algues rencontrées au niveau spécifique, afin de mieux définir les indicateurs algaux de pollution. De même, les facteurs environnementaux tels que les ions majeurs, les nutriments, l'oxygène dissous et la demande biochimique en oxygène devront être pris en compte lors des prochaines études afin de mieux cerner les paramètres qui contrôlent la variabilité spatio-temporelles des assemblages.

\section{REMERCIEMENTS}

Les auteurs remercient les experts anonymes pour les multiples critiques qui ont permis d'améliorer la qualité du manuscrit. Ils tiennent également à remercier $\mathrm{M}^{\mathrm{me}}$ Bernadette NOUMSSI pour son aide dans l'identification des taxons d'algues rencontrés.

\section{RÉFÉRENCES BIBLIOGRAPHIQUES}

AMOUGOU A., E.R. ATANGANA, N.I.M. KENGNE, T FONKOU, J. NYA et E.A. NDIKEFOR (1999). Programme fédérateur de recherche sur l'assainissement des eaux usées en Afrique sub-saharienne - volet lagunage. Rapport de recherche du programme CAMPUS. Financement de la coopération française, Imputation, 94016400.06. M229.0040, 27 p.

BATTARBEE R.W. et D.F. CHARLES (1986). Diatombased $\mathrm{pH}$ reconstruction studies of acid lakes in Europe and North America: A synthesis. Wat. Air Soil Pollut., 30, 374-354.

BARKER P.A., N. ROBERT, H.F. LAMB, S. VAN DER KAARS et A. BENKADDOUR (1994). Interpretation of Holocene lake-level change from diatom assemblages in Lake Sidi Ali, Middle Atlas, Morocco. J. Paleolimnol., 12, 223-234.

BONNARD R., M. LAFONT et P. LE PIMPEC (2003). Notions d'hydro-écologie et de qualité biologique des eaux courantes. Ingénieries, 33, 3-12.

BOURRELLY P. (1984). Algues d'eau douce de la Nouvelle-Calédonie recueillies par la Mission F. Starmühlneren 1965 (Diatomées exclues). 2 $2^{\mathrm{e}}$ Partie: Chlorophycées (Desmidiées) et Charophycées (1). Rev. Hydrobiol. Trop., 17, 101-115.

BOURRELLY P. et E. MANGUIN (1952). Algues d'eau douce de la Guadeloupe. SEDES (Éditeur), France, 276 p.

BRUGAM R.B., K. MC KEEVER et L. KOLESA (1998). A diatom-inferred water depth reconstruction for an Upper Peninsula, Michigan, Lake. J. Paleolimnol., 20, 267-276.

CHARLES D.F., S.S. DIXIT, B.F. CUMMING et J.P. SMOL (1991). Variability in diatom and Chrysophytes assemblages and inferred $\mathrm{pH}$ : paleolimnological studies of Big Moose Lake, New York, USA. J. Paleolimnol., 5, 267-284.

COMPÈRE P. (1974). Algues de la région du Tchad. II Cyanophycées. Cah. ORSTOM Ser. Hydrobiol., VIII, 165-198.

COMPÈRE P. (1976). Algues de la région du Tchad. VI- Chlorophycophytes ( $2^{\mathrm{e}}$ partie: Ulotrichophycées, Zygnématacées) (1). Cah. ORSTOM Ser. Hydrobiol., X, 135-164.

COMPÈRE P. (1977). Algues de la région du Tchad. VIIChlorophycophytes (3 $3^{\text {e }}$ Partie: Desmidiées) (1). Cah. 
ORSTOM Ser. Hydrobiol., XI, 77-177.

COUTÉ A. et G. ROUSSELIN (1975). Contribution à l'étude des algues d'eau douce du moyen Niger (Mali). Bull. Mus. Nat. Hist. Nat. Paris Ser. Bot., 21, 73-176.

DAUTA A. et J. FEUILLADE (1995). Croissance et dynamiques des populations algales. Dans: Limnologie générale (sous la direction de R. Pourriot et M. Meybeck), MASSON, pp. 328-350.

FOLEFACK C.A. (1989). Étude de la flore algale du lac municipal de Yaoundé. Mémoire de Maîtrise, Univ. Yaoundé, 77 p.

FONKOUT., V.F. NGUETSOP,J.Y.PINTA, V.M. DEKOUM, M. LEKEUFACK et A. AMOUGOU (2005). Macrophyte diversity in polluted and non-polluted wetlands in Cameroon. Cameroon J. Exper. Biol., 01, 26-33.

GASSE F. (1980). Les diatomées lacustres plio-pléistocène du Gadeb (Ethiopie). Systématique, paléoécologie, biostratigraphie. Rev. Algol., 249 p.

GASSE F. (1986). Diatom assemblages in East Africa: classification, distribution and ecology. Rev. Hydrobiol. Trop., 16, 3-34.

GASSE F. (2002). Diatom-inferred salinity and carbonate oxygen isotopes in Holocene waterbodies of western Sahara and Sahel (Africa). Quat. Sci. Rev., 21, 737-767.

$\mathrm{HACH}$ (1997). Water analysis handbook. $3^{\text {rd }}$ edition, LOVELAND (Éditeur), Colorado. 1309 p.

HUSTEDT F. (1930). Die Süsswasser-Flora Mitteleuropas. Bacillariophyta (Diatomeae). A. PASCHER (Éditeur), Verlag Von Gustav Fischer, Jena, Allemagne de l'Est, $466 \mathrm{p}$.

ILTIS A. (1980). Les algues. Dans: Flore et Faune aquatiques de l'Afrique sahelo-soudanienne. J.R. DURAND et C. LEVÊQUE (Éditeurs), ORSTOM; t1, pp. 9-61.

IWONA J. (1995). The influence of macrophytes on a phytoplankton community in experimental conditions. Hydrobiol., 306, 21-32.

KEMKA N., T. NJINE, T.S.H. ZÉBAZÉ, D. NIYITEGEKA, M. NOLA, A. MONKIEDJE, J. DEMANOU et M.S. FOTO (2004). Phytoplancton du lac municipal de Yaoundé (Cameroun) : succession écologique et structure des peuplements. Rev. Sci. Eau, 17, 301-316.
KEMKA N., T. NJINE, T.S.H. ZÉBAZÉ, M.S. FOTO, M. NOLA, A. MONKIEDJE, D. NIYITEGEKA et P. COMPÈRE (2006). Eutrophication of lakes in urbanized areas: The case of Yaoundé Municipal Lake in Cameroon, Central Africa. Lake Reservoir Research Manage., 11, $47-55$.

KRAMMER K. et H. LANGE-BERTALOT (1986). Süsswasserflora von Mitteleuropa. Bacillariophyceae. Teil 1: Naviculaceae. G Fischer Verlag, Stuttgart, Allemagne, $876 \mathrm{p}$.

KRAMMER K. et H. LANGE-BERTALOT (1988). Süsswasserflora von Mitteleuropa. Bacillariophyceae. Teil 2: Bacillariaceae, Epithemiaceae, Surirellaceae. G. Fischer Verlag, Stuttgard, Allemagne, 596 p.

KRAMMER K. et H. LANGE-BERTALOT (1991). Süsswasserflora von Mitteleuropa. Bacillariophyceae. Teil 3: Centrales, Fragilariaceae, Eunotiaceae. G. Fischer Verlag, Stuttgart, Allemagne, $576 \mathrm{p}$.

LUDES B. et M. COSTE (1996). Diatomées et médecine légale. Tec \& Doc. Lavoisier, France, Éditions Médicales Internationales, $258 \mathrm{p}$.

MISKANE N. (1997). Les paléo-environnements de haute montagne pendant l'Holocène récent d'après l'étude des diatomées actuelles et fossiles de deux vallées glaciaires de Bolivie. Implication paléoclimatique. Thèse de Doctorat, Musée National d'Histoire, Paris, 386 p.

NGUETSOP V. F. (1990). Flore algale de quelques marécages à Cyrtosperma senegalense dans le région de Yaoundé. Mémoire de Maîtrise, Univ. Yaoundé, 88 p.

NGUETSOP V.F., T. FONKOU, V.M. DEKOUM, M.N. NDEMAFO et J.Y. PINTA (2007). Relationships between algae taxa and physico-chemical characteristics of water in wetlands and waterbodies. Cameroon J. Exper. Biol., 03, 70-79.

NGUETSOP V.F., S. SERVANT-VILDARY et M. SERVANT (2004). Late Holocene climatic changes in West Africa, a high resolution diatom record from equatorial Cameroon, Quat. Sci. Rev., 23, 591-609.

PRYGIEL J. et M. COSTE (1993). Utilisation des indices diatomiques pour la mesure de la qualité des eaux du bassin Artois-Picardie : bilan et perspectives. Ann. Limnol., 29, 255-267. 
SERVANT-VILDARY S. et M. ROUX (1990). Multivariate analysis of diatoms and water chemistry in Bolivian saline lakes. Hydrobiol., 197, 167-290.

SLADECEK V. (1986). Diatoms as indicators of organic pollution. Acta Hydrochim. Hydrobiol., 14, 555-566.

STAGER J.C. et R. ANFANG-SUTTER (1999). Preliminary evidence of environmental changes at Lake Bambili (Cameroon, West Africa) since 24,000 BP. J. Paleolimnol., 22, 319-330.

TESTARD P. (1995). Rôle des macrophytes littoraux dans le fonctionnement des écosystèmes lacustres. Dans: Limnologie générale (sous la direction de R. Pourriot et M. Meybeck), Masson, pp. 296-326.

TRÉMOLIÈRES M. (2004). Fiche habitat 3150: Lacs eutrophes naturels avec végétation du Magnopotamion ou Hydrocharition. Dans : Reférentiel des habitats reconnus d'intérêt communautaire de la bande rhénane: Description, état de conservation et mesures de gestion. Conservatoire des sites alsaciens et office national des forêts, pp. 83-94.

USEPA (2003). Biological indicators of watershed health: periphyton as indicators. www.epa.gov/owow/wetlands/ wqual/algae.html. (Consultation le 21 avril 2006). 